\title{
Mesure invariante pour le système d'équations stochastiques du modèle de compétition avec diffusion spatiale
}

\author{
Saliha Hamdous $(*)$ - Hisao Fujita Yashima (**)
}

\section{Introduction.}

Depuis quelques années la question de la mesure invariante est devenue un des thèmes centraux de la recherche sur les équations stochastiques de la dynamique de populations. En particulier, Rudnicki [14] a démontré l'existence et l'unicité de la mesure invariante pour l'équation stochastique du modèle proie-prédateur, en précisant les conditions sur les coefficients de l'équation pour l'existence de la mesure invariante et l'extention de son support. D'autre part, pour le modèle stochastique de compétition entre deux espèces, dans [16] le comportement asymptotique de la solution de l'équation stochastique, y compris le cas de convergence vers une mesure invariante, a été classifié, en utilisant la méthode de Rudnicki et l'application directe de la fonction de Khas'minskii (voir [10]). Or, dans le cas où on considère aussi la diffusion spatiale de la population dans un territoire, Tornatore [15] a démontré que pour $n$ espèces en compétition l'espérance mathématique des populations totales, qui résultent de l'équation stochastique, est uniformément bornée pour tout $t \geq 0$. Dans [7] nous avons démontré l'existence d'une mesure invariante pour un système d'équations analogue à celui de [15] mais avec une compétition limitée entre les espèces différentes.

Le but du présent travail est de démontrer que l'équation stochastique pour deux espèces en compétition dans la forme usuelle avec la diffusion

(*) Indirizzo dell'A.: Département de Mathématiques, Université M. Mammeri, 15000, Tizi-Ouzou, Algérie, et Dipartimento di Matematica, Università di Torino, 10123 Torino, Italia.

E-mail: hamdoussaliha2002@yahoo.fr, saliha.hamdous@unito.it

(**) Indirizzo dell'A.: Dipartimento di Matematica, Università di Torino, 10123 Torino, Italia.

E-mail: fujitayashima@unito.it 
spatiale admet, sous une condition convenable et naturelle, une mesure invariante, pour laquelle aucune des deux espèces n'est destinée à l'extinction.

Pour les modèles de la dynamique de populations en général et ceux avec la diffusion spatiale ou des perturbations stochastiques, voir aussi [17], [12], [3], [8], [1], [2], [6] et d'autres.

\section{Résultat principal.}

Considérons un système écologique formé par deux espèces végétales ou animales dans un territoire $D$ en compétition pour des ressources du territoire. Pour formuler le problème dans une forme mathématique, admettons que $D$ soit un ensemble ouvert borné de $\boldsymbol{R}^{d}, d=2$ ou 3 , muni de la frontière régulière $\partial D$ et désignons par $N_{1}(t, x)$ et $N_{2}(t, x)$ la densité de population au point $x \in D$ et à l'instant $t \in \boldsymbol{R}$ de la première et de la seconde espèce respectivement. Cela étant, nous considérons le système d'équations stochastiques

(2.1) $d N_{i}(t, \cdot)$

$=\left[\left(\alpha_{i}-\sum_{j=1}^{2} \beta_{i j} N_{j}(t, \cdot)\right) N_{i}(t, \cdot)+\varepsilon_{i} \Delta N_{i}(t, \cdot)\right] d t+\rho_{i} N_{i}(t, \cdot) d W(t), \quad i=1,2$,

dans l'espace de Hilbert $L^{2}(D)$ avec la condition aux limites

$$
\nabla N_{i} \cdot \vec{n}=0 \quad \text { sur } \partial D, \quad i=1,2 ;
$$

dans (2.1)-(2.2) l'opérateur de Laplace $\Delta$ et le nabla $\nabla$ sont à considérer par rapport aux variables spatiales $x_{1}, \cdots, x_{d}, \vec{n}$ désigne le vecteur normal à la frontière $\partial D$ de $D$ et les coefficients $\alpha_{i}, \beta_{i j}, \varepsilon_{i}$ et $\rho_{i}(i, j=1,2)$ sont des constantes telles que

$$
\alpha_{i}>0, \quad \varepsilon_{i}>0, \quad \rho_{i}>0, \quad \beta_{i i}>0, \quad i=1,2, \quad \beta_{i j} \geq 0, \quad i, j=1,2 \quad i \neq j,
$$

tandis que $W(t)$ est un mouvement brownien défini sur une base stochastique

$$
\left(\Omega, \mathcal{F},\left(\mathcal{F}_{t}\right)_{t \geq 0}, \boldsymbol{P}\right)
$$

à valeurs dans $L^{2}(D)$. On suppose que $W(t)$ est de la forme

$$
W(t)=\sum_{m=1}^{\infty} \lambda_{m} e_{m}(\cdot) W^{(m)}(t)
$$

où $\left\{e_{m}\right\}_{m=1}^{\infty}$ est une base orthonormale de $L^{2}(D)$ avec $e_{m}(\cdot) \in L^{\infty}(D)$ pour 
tout $m \in N \backslash\{0\}, W^{(m)}(t), m=1,2, \cdots$, sont des mouvements browniens indépendants à valeurs réelles, et $\left\{\lambda_{m}\right\}_{m=1}^{\infty}$ est une suite de nombres réels telle que

$$
\left\|\sum_{m=1}^{\infty} \lambda_{m}^{2} e_{m}^{2}(\cdot)\right\|_{L^{\infty}(D)}=K_{0}<\infty .
$$

Pour le problème (2.1)-(2.2) proposé ci-dessus, on connaît l'existence et l'unicité ainsi que la positivité de la solution. Plus précisément, on a la

Proposition 2.1. Le problème (2.1)-(2.2) avec la condition initiale $N_{i}(0, x)>0$ p.p. dans $D, N_{i}(0, \cdot) \in L^{2}(D), i=1,2$, admet une solution $N(t, x)=\left(N_{1}(t, x), N_{2}(t, x)\right)$ et une seule dans l'intervalle $0 \leq t<\infty$ et on a $N_{i}(t, x)>0$ p.p. dans $D$ p.s. $(i=1,2)$ pour tout $t \geq 0$.

Pour la démonstration, voir [15] (dans [15] l'existence et l'unicité de la solution sont démontrées pour un système de $n$ espèces).

Pour démontrer l'existence d'une mesure invariante, nous supposons que, avec $K_{0}$ défini dans (2.4)-(2.5), on ait

$$
\frac{\beta_{21}}{\beta_{11}}<\frac{\alpha_{2}-K_{0} \frac{\rho_{1}^{2}+\rho_{2}^{2}}{2}}{\alpha_{1}} \leq \frac{\alpha_{2}}{\alpha_{1}-K_{0} \frac{\rho_{1}^{2}+\rho_{2}^{2}}{2}}<\frac{\beta_{22}}{\beta_{12}},
$$

où on entend $\frac{\beta_{22}}{\beta_{12}}=+\infty$ si $\beta_{12}=0$. Comme $\frac{\beta_{21}}{\beta_{11}} \geq 0$ et que $\alpha_{i}>0, i=1,2$, l'inégalité (2.6) implique que

$$
\alpha_{i}-K_{0} \frac{\rho_{i}^{2}}{2} \geq \alpha_{i}-K_{0} \frac{\rho_{1}^{2}+\rho_{2}^{2}}{2} 0, \quad i=1,2,
$$

Notre résultat principal est le suivant.

THÉORÈme 2.1. Si les coefficients $\alpha_{i}, \beta_{i j}$ et $\rho_{i}(i, j=1,2)$ et le mouvement brownien $W(t)$ satisfont aux conditions (2.3)-(2.6), alors il existe une mesure invariante $\bar{\mu}$ dans $L^{2}\left(D ; \boldsymbol{R}^{2}\right)$ pour l'équation (2.1) avec la condition aux limites (2.2), telle que

$$
\begin{aligned}
\bar{\mu}\left(\left\{N_{i} \in H^{1}(D), i=1,2\right\}\right) & =\bar{\mu}\left(\left\{N_{i}>0 \text { p.p. dans } D, i=1,2\right\}\right) \\
& =\bar{\mu}\left(\left\{\log N_{i} \in L^{1}(D), i=1,2\right\}\right)=1 .
\end{aligned}
$$

La population totale de l'espèce $i$ sur le territoire $D$, qui est essentielle du point de vue biologique, est exprimée exactement par la norme 
$\left\|N_{i}\right\|_{L^{1}(D)}$. Mais pour les traitements mathématiques il nous est commode de considérer $N_{i}$ comme élément de $L^{2}(D)$; comme on voit facilement, la norme $\left\|N_{i}\right\|_{L^{1}(D)}$ est majorée par $\sqrt{m e s(D)}\left\|N_{i}\right\|_{L^{2}(D)}$.

\section{Démonstration de l'existence d'une mesure invariante.}

La démonstration du théorème 2.1 se base sur l'application du théorème de Krylov-Bogoliubov, qui, à son tour, s'appuie sur une estimation de la solution du problème (2.1)-(2.2) avec les conditions initiales et utilise également les idées du théorème de Khas'minskii (voir [10]). Comme l'estimation de la solution qu'on va utiliser est assez complexe, en renvoyant sa démonstration au paragraphe suivant, dans le présent paragraphe on va démontrer le théorème 2.1 en admettant le résultat de l'estimation.

Pour formuler ce résultat auxiliaire, on pose

$$
\begin{gathered}
\Phi_{0}(N)=\sum_{i=1}^{2}\left(\left\|N_{i}\right\|_{L^{2}(D)}^{2}+\left\|\log N_{i}\right\|_{L^{1}(D)}\right), \\
\Phi_{1}(N)=\sum_{i=1}^{2}\left(\left\|\nabla N_{i}\right\|_{L^{2}(D)}^{2}+\left\|\nabla \log N_{i}\right\|_{L^{2}(D)}^{2}\right), \\
\Xi(D)=\left\{N \in L^{2}\left(D ;\left(\boldsymbol{R}_{+}\right)^{2}\right) \mid \log N_{i} \in L^{1}(D), i=1,2\right\} .
\end{gathered}
$$

On a alors le lemme suivant.

LEMme 3.1. Il existe une fonction $G: \Xi(D) \rightarrow \boldsymbol{R} \cup\{-\infty\}$ telle que, si on pose

$$
A(c)=\sup \left\{G(N) \mid N \in \Xi(D), \Phi_{0}(N) \geq c\right\},
$$

on ait

$$
\begin{gathered}
\sup _{N \in \Xi(D)} G(N)=A(0)<\infty \\
A(c) \rightarrow-\infty \quad \text { pour } c \rightarrow+\infty,
\end{gathered}
$$

et que de plus, si $N(t, \cdot)$ est la solution du problème (2.1)-(2.2) avec la condition initiale $N(0, \cdot)=N_{0} \in \Xi(D)$, alors on ait

$$
\kappa_{0} \boldsymbol{E} \Phi_{0}(N(t, \cdot))+\kappa_{1} \int_{0}^{t} \boldsymbol{E} \Phi_{1}\left(N\left(t^{\prime}, \cdot\right)\right) d t^{\prime} \leq \kappa_{2}\left\|N_{0}\right\|_{\Xi}^{2}+\int_{0}^{t} \boldsymbol{E} G\left(N\left(t^{\prime}, \cdot\right)\right) d t^{\prime}
$$


où $\kappa_{0}, \kappa_{1}, \kappa_{2}$ sont des constantes positives et

$$
\|N\|_{\Xi}=\sum_{i=1}^{2}\left(\left\|N_{i}\right\|_{L^{2}(D)}+\left\|\log N_{i}\right\|_{L^{1}(D)}\right)
$$

En renvoyant la démonstration de ce lemme au paragraphe 4, on expose ici la démonstration du théorème 2.1.

DÉmonstration DU THÉORÈme 2.1. Comme le premier membre de (3.7) est non-négatif, on a

$$
0 \leq \kappa_{2}\left\|N_{0}\right\|_{\Xi}^{2}+t A(0)+A(c) \int_{0}^{t} \boldsymbol{P}\left(\left\{\Phi_{0}\left(N\left(t^{\prime}, \cdot\right)\right) \geq c\right\}\right) d t^{\prime} ;
$$

en particulier pour $c$ tel que $A(c)<0$, on a

$$
\frac{1}{t} \int_{0}^{t} \boldsymbol{P}\left(\left\{\Phi_{0}\left(N\left(t^{\prime}, \cdot\right)\right) \geq c\right\}\right) d t^{\prime} \leq \frac{1}{-A(c)}\left(A(0)+\frac{\kappa_{2}\left\|N_{0}\right\|_{\Xi}^{2}}{t}\right) .
$$

D’autre part, de (3.7) on déduit immédiatement que

$$
\kappa_{1} \frac{1}{t} \int_{0}^{t} \boldsymbol{E} \Phi_{1}\left(N\left(t^{\prime}, \cdot\right)\right) d t^{\prime} \leq A(0)+\frac{\kappa_{2}\left\|N_{0}\right\|_{\Xi}^{2}}{t} .
$$

De (3.9)-(3.10) et de (3.6) on déduit

$$
\frac{1}{t} \int_{0}^{t} \boldsymbol{P}\left(\left\{\Phi_{0}\left(N\left(t^{\prime}, \cdot\right)\right)+\Phi_{1}\left(N\left(t^{\prime}, \cdot\right)\right) \leq c\right\}\right) d t^{\prime} \geq 1-\varepsilon(c) \quad \forall t \geq 1
$$

avec une fonction décroissante $\varepsilon(c)$ telle que

$$
\varepsilon(c) \rightarrow 0 \quad \text { pour } \quad c \rightarrow \infty
$$

Pour $N_{0} \in \Xi(D)$ et un ensemble borélien $\Gamma$ de $L^{2}\left(D ; \boldsymbol{R}^{2}\right) \times L^{1}\left(D ; \boldsymbol{R}^{2}\right)$, on pose

$$
P_{t}\left(N_{0}, \Gamma\right)=\boldsymbol{P}\left(\left\{\left(N_{1}(t, \cdot), N_{2}(t, \cdot), \log N_{1}(t, \cdot), \log N_{2}(t, \cdot)\right) \in \Gamma\right\}\right),
$$

où $N(t, \cdot)$ est la solution du problème (2.1)-(2.2) avec la condition initiale

$$
N_{i}(0, x)=N_{0, i}(x), \quad i=1,2 .
$$


On définit alors

$$
v_{T}(\Gamma)=\frac{1}{T} \int_{0}^{T} P_{t}\left(N_{0}, \Gamma\right) d t
$$

Cela étant, de (3.11) et de la définition de $\Phi_{0}(\cdot)$ et $\Phi_{1}(\cdot)$ (voir (3.1) et (3.2)) on déduit qu'on a

$$
v_{T}\left(\left\{N \in \Xi(D) \mid\|N\|_{H^{1}\left(D ; \boldsymbol{R}^{2}\right)}^{2}+\sum_{i=1}^{2}\left\|\log N_{i}\right\|_{H^{1}(D ; \boldsymbol{R})}^{2} \leq c\right\}\right) \geq 1-\tilde{\varepsilon}(c) \quad \forall T \geq 1
$$

avec une fonction décroissante $\tilde{\varepsilon}(\cdot)$ telle que

$$
\tilde{\varepsilon}(c) \rightarrow 0 \text { pour } \quad c \rightarrow \infty .
$$

C'est-à-dire, pour tout $\varepsilon>0$ il existe un nombre $C_{(\varepsilon)}$ tel que

$$
\begin{aligned}
v_{T}\left(\left\{N \in \Xi(D) \mid\|N\|_{H^{1}\left(D ; \boldsymbol{R}^{2}\right)}^{2}+\sum_{i=1}^{2}\left\|\log N_{i}\right\|_{H^{1}(D ; \boldsymbol{R})}^{2} \leq C_{(\varepsilon)}\right\}\right) & \geq 1-\varepsilon, \\
& \forall T \geq 1 .
\end{aligned}
$$

On rappelle qu'un ensemble borné dans $H^{1}\left(D ; \boldsymbol{R}^{2}\right) \times H^{1}\left(D ; \boldsymbol{R}^{2}\right)$ est relativement compact dans $L^{2}\left(D ; \boldsymbol{R}^{2}\right) \times L^{1}\left(D ; \boldsymbol{R}^{2}\right)$. Donc en vertu du théorème de Krylov-Bogoliubov ([11]) dans sa version complétée par le critère de Prokhorov (voir [4], [5]), on déduit de (3.12) l'existence d'une mesure invariante $\bar{v}$ dans $L^{2}\left(D ; \boldsymbol{R}^{2}\right) \times L^{1}\left(D ; \boldsymbol{R}^{2}\right)$ pour l'équation (2.1) avec la condition aux limites (2.2). Désignons par $\mu_{T}$ et $\lambda_{T}$ les projections de $v_{T}$ sur $L^{2}\left(D ; \boldsymbol{R}^{2}\right)$ et sur $L^{1}\left(D ; \boldsymbol{R}^{2}\right)$ et analoguement par $\bar{\mu}$ et $\bar{\lambda}$ les projections de $\bar{v}$ sur $L^{2}\left(D ; \boldsymbol{R}^{2}\right)$ et sur $L^{1}\left(D ; \boldsymbol{R}^{2}\right)$ respectivement. Or, comme $\bar{v}$ est obtenue comme limite faible d'une suite $\left\{v_{T_{k}}\right\}_{k=1}^{\infty}$ (voir [4]; voir aussi le théorème 1 du $§ 1$, chap. IX de [9]) et que

$$
\mu_{T_{k}}\left(\left\{N_{i}>0 \text { p.p. dans } D, i=1,2\right\}\right)=1, \quad \lambda_{T_{k}}=\log \circ \mu_{T_{k}},
$$

on a

$$
\bar{\mu}\left(\left\{N_{i}>0 \text { p.p. dans } D, i=1,2\right\}\right)=1, \quad \bar{\lambda}=\log \circ \bar{\mu},
$$

ce qui achève la démonstration du théorème 2.1

\section{Démonstration du lemme 3.1.}

Pour démontrer le lemme 3.1, on va appliquer la formule d'Ito à une fonction convenable et en obtenir une estimation, en utilisant les idées de la 
fonction de Khas'minskii et celles des estimations dans $L^{2}(D)$ de la solution de l'équation (2.1) avec la condition (2.2) (voir [15]).

Commençons par remarquer une conséquence élémentaire de la condition (2.6).

Remarque 4.1. Pour $i=1,2$, il existe $k_{i}>0$ tel que

$$
\left(\beta_{i i}+\beta_{j i}+k_{i} \alpha_{i}\right)^{2}-4 k_{i} \beta_{i i}\left(\alpha_{1}+\alpha_{2}-K_{0} \frac{\rho_{1}^{2}+\rho_{2}^{2}}{2}\right)<0, \quad j \neq i .
$$

DÉmonstration. En considérant (4.1) comme équation algébrique du second degré en $k_{i}$, on voit qu'il existe $k_{i}>0$ satisfaisant à (4.1), si et seulement si

$$
\begin{gathered}
\alpha_{i}\left(\beta_{i i}+\beta_{j i}\right)-2 \beta_{i i}\left(\alpha_{1}+\alpha_{2}-K_{0} \frac{\rho_{1}^{2}+\rho_{2}^{2}}{2}\right)<0 \\
\left(\alpha_{i}\left(\beta_{i i}+\beta_{j i}\right)-2 \beta_{i i}\left(\alpha_{1}+\alpha_{2}-K_{0} \frac{\rho_{1}^{2}+\rho_{2}^{2}}{2}\right)\right)^{2}-\alpha_{i}^{2}\left(\beta_{i i}+\beta_{j i}\right)^{2}>0, \quad j \neq i .
\end{gathered}
$$

On voit aisément que, pour que ces deux inégalités soient vérifiées, il faut et il suffit que

$$
\alpha_{i}\left(\beta_{i i}+\beta_{j i}\right)<\beta_{i i}\left(\alpha_{1}+\alpha_{2}-K_{0} \frac{\rho_{1}^{2}+\rho_{2}^{2}}{2}\right), \quad j \neq i .
$$

Cette dernière inégalité pour $i=1$ et 2 est équivalente à (2.6).

On pose maintenant

$$
U(N)=\sum_{i=1}^{2}\left(k_{i} N_{i}-\log N_{i}\right)+C,
$$

où $k_{1}$ et $k_{2}$ sont deux constantes positives satisfaisant à (4.1) et

$$
C=1-\inf _{N \in\left(\boldsymbol{R}_{+}\right)^{2}} \sum_{i=1}^{2}\left(k_{i} N_{i}-\log N_{i}\right) .
$$

Grâce à ce choix de $C$, on a

$$
U(N) \geq 1 \quad \forall N \in\left(\boldsymbol{R}_{+}\right)^{2}
$$

et il existe des constantes positives $\bar{c}_{1}, \bar{c}_{2}, \bar{c}_{3}, \bar{c}_{4}$ telles que

$$
\bar{c}_{1} \sum_{i=1}^{2}\left|\log N_{i}\right| \leq U(N) \leq \bar{c}_{2}+\bar{c}_{3} \sum_{i=1}^{2}\left|\log N_{i}\right|+\bar{c}_{4} \sum_{i=1}^{2}\left|N_{i}\right| .
$$


On pose en outre

$$
\varphi(N)=\frac{1}{2} \sum_{i=1}^{2} \int_{D} N_{i}^{2} d x+\frac{1}{2}\left(\int_{D} U\left(N_{1}, N_{2}\right) d x\right)^{2} .
$$

On va appliquer la formule d'Ito à la fonction $\varphi(N)$. Pour cela, rappelons que

$$
\begin{gathered}
\frac{\partial \varphi(N)}{\partial N_{i}}(f)=\int_{D} N_{i} f d x+\int_{D} U(N) d x \int_{D} \frac{\partial U(N)}{\partial N_{i}} f d x, \\
\frac{\partial^{2} \varphi(N)}{\partial N_{i} \partial N_{j}}(f)(g)=\delta_{i j} \int_{D} f g d x+\int_{D} U(N) d x \int_{D} \frac{\partial^{2} U(N)}{\partial N_{i} \partial N_{j}} f g d x \\
+\int_{D} \frac{\partial U(N)}{\partial N_{j}} f d x \int_{D} \frac{\partial U(N)}{\partial N_{i}} g d x, \\
\frac{\partial U(N)}{\partial N_{i}}=k_{i}-\frac{1}{N_{i}}, \quad \frac{\partial^{2} U(N)}{\partial N_{i} \partial N_{j}}=\frac{\delta_{i j}}{N_{i}^{2}} .
\end{gathered}
$$

En appliquant la formule d'Ito à la fonction $\varphi(N)$ (pour la formule d'Ito pour les processus à valeurs dans un espace de Hilbert, voir par exemple [13]) et en intégrant par parties sur $D$ les termes contenant l'opérateur de Laplace 4 , on obtient

$$
\begin{aligned}
\varphi(N(t, \cdot)) & +\int_{0}^{t} F\left(N\left(t^{\prime}, \cdot\right)\right) d t^{\prime}=\varphi(N(0, \cdot)) \\
& +\int_{0}^{t}\left(G_{1}\left(N\left(t^{\prime}, \cdot\right)\right)+G_{2}\left(N\left(t^{\prime}, \cdot\right)\right)+G_{3}\left(N\left(t^{\prime}, \cdot\right)\right)\right) d t^{\prime} \\
& +\int_{0}^{t}\left\langle h\left(N\left(t^{\prime}, \cdot\right)\right), d W\left(t^{\prime}\right)\right\rangle,
\end{aligned}
$$

où

$$
\begin{gathered}
F(N)=\sum_{i=1}^{2} \varepsilon_{i}\left(\left\|\nabla N_{i}\right\|_{L^{2}(D)}^{2}+\frac{1}{2}\|U(N)\|_{L^{1}(D)}\left\|\nabla \log N_{i}\right\|_{L^{2}(D)}^{2}\right), \\
G_{1}(N)=\sum_{i=1}^{2} \int_{D} N_{i}^{2}\left(\alpha_{i}+\frac{\rho_{i}^{2}}{2} \sum_{m=1}^{\infty} \lambda_{m}^{2} e_{m}^{2}-\sum_{j=1}^{2} \beta_{i j} N_{j}\right) d x
\end{gathered}
$$


$(4.9)$

(4.10) $A U(N)=\sum_{i=1}^{2} \frac{\partial U}{\partial N_{i}}\left(\alpha_{i}-\sum_{j=1}^{2} \beta_{i j} N_{j}\right) N_{i}+\sum_{i, j=1}^{2} \frac{\rho_{i} \rho_{j}}{2} N_{i} N_{j} \frac{\partial^{2} U}{\partial N_{i} \partial N_{j}} \sum_{m=1}^{\infty} \lambda_{m}^{2} e_{m}^{2}$

$$
\begin{gathered}
G_{3}(N)=\frac{1}{2} \sum_{m=1}^{\infty} \sum_{i, j=1}^{2} \int_{D} \frac{\partial U}{\partial N_{i}} \rho_{i} N_{i} \lambda_{m} e_{m} d x \int_{D} \frac{\partial U}{\partial N_{j}} \rho_{j} N_{j} \lambda_{m} e_{m} d x \\
h(N)=\sum_{i=1}^{2} \rho_{i} N_{i}^{2}+\int_{D} U d x \sum_{i=1}^{2} \frac{\partial U}{\partial N_{i}} \rho_{i} N_{i}
\end{gathered}
$$

LEMME 4.1. On $a$

$$
\sup _{N \in \Xi(D)} G_{2}(N)<\infty
$$

$$
\sup \left\{G_{2}(N) \mid N \in \Xi(D), \int_{D} U(N) d x \geq c\right\} \rightarrow-\infty \text { pour } c \rightarrow+\infty
$$

DÉmonstration. En substituant les expressions de $\frac{\partial U(N)}{\partial N_{i}}$ et de $\frac{\partial^{2} U(N)}{\partial N_{i} \partial N_{j}}$ dans (4.10), on obtient

$$
A U(N)=\gamma(N)
$$

$$
\begin{aligned}
\gamma(N)= & -\left(k_{1} N_{1}-1\right) \beta_{11} N_{1}+\left(\beta_{21}+k_{1} \alpha_{1}\right) N_{1}-\left(k_{1} \beta_{12}+k_{2} \beta_{21}\right) N_{1} N_{2} \\
& -\left(k_{2} N_{2}-1\right) \beta_{22} N_{2}+\left(\beta_{12}+k_{2} \alpha_{2}\right) N_{2}-\alpha_{1}-\alpha_{2} \\
& +\frac{\rho_{1}^{2}+\rho_{2}^{2}}{2} \sum_{m=1}^{\infty} \lambda_{m}^{2} e_{m}^{2}
\end{aligned}
$$

On remarque d'abord que

$$
\gamma(N) \rightarrow-\infty \quad \text { pour } \quad|N|^{2}=N_{1}^{2}+N_{2}^{2} \rightarrow \infty
$$

et donc

$$
\sup _{N \in\left(\boldsymbol{R}_{+}\right)^{2}} \gamma(N) \equiv \bar{\Gamma}<\infty
$$


d'où, compte tenu de (4.15), il résulte que

$$
\sup _{N \in \Xi(D)}\left(\int_{D} A U(N) d x-\frac{1}{2} \sum_{i=1}^{2} \varepsilon_{i}\left\|\nabla \log N_{i}\right\|_{L^{2}(D)}^{2}\right)<\infty .
$$

On va démontrer qu'il existe deux constantes positives $\varepsilon_{G}$ et $L_{G}$ telles que

$$
\gamma(N) \leq-\varepsilon_{G} \quad \text { pour } \quad \sum_{i=1}^{2}\left|\log N_{i}\right| \geq L_{G}
$$

Pour ce faire, étant donné qu'on a (4.17), il suffit de démontrer qu'il existe deux constantes positives $\varepsilon_{G}$ et $\delta_{G}$ telles que

$$
\gamma(N) \leq-\varepsilon_{G} \quad \text { pour } \min \left(N_{1}, N_{2}\right) \leq \delta_{G},
$$

ou, compte tenu de la continuité de $\gamma(N)$ en $N_{1}$ et $N_{2}$ (voir (4.11)), de démontrer que

$$
\sup _{N_{i}>0} \psi_{i}\left(N_{i}\right)<0, \quad i=1,2, \quad \psi_{i}\left(N_{i}\right)=\left.\gamma(N)\right|_{N_{j}=0}, \quad j \neq i .
$$

En tenant compte de la condition (2.5), de l'expression (4.16) de $\gamma(N)$ on déduit que

$\psi_{i}\left(N_{i}\right)=-\beta_{i i} k_{i} N_{i}^{2}+\left(\beta_{i i}+\beta_{j i}+k_{i} \alpha_{i}\right) N_{i}-\alpha_{1}-\alpha_{2}+\frac{\rho_{1}^{2}+\rho_{2}^{2}}{2} \sum_{m=1}^{\infty} \lambda_{m}^{2} e_{m}^{2} \leq \tilde{\psi}_{i}\left(N_{i}\right)$, où

$$
\tilde{\psi}_{i}\left(N_{i}\right)=-\beta_{i i} k_{i} N_{i}^{2}+\left(\beta_{i i}+\beta_{j i}+k_{i} \alpha_{i}\right) N_{i}-\alpha_{1}-\alpha_{2}+K_{0} \frac{\rho_{1}^{2}+\rho_{2}^{2}}{2} .
$$

Il est clair que pour avoir

$$
\sup _{N_{i}>0} \tilde{\psi}_{i}\left(N_{i}\right)<0
$$

il faut et il suffit qu'on ait

$$
\Delta=\left(\beta_{i i}+\beta_{j i}+k_{i} \alpha_{i}\right)^{2}-4 \beta_{i i}\left(\alpha_{1}+\alpha_{2}-K_{0} \frac{\rho_{1}^{2}+\rho_{2}^{2}}{2}\right) k_{i}<0, \quad j \neq i .
$$

Or, l'existence d'un $k_{i}>0$ satisfaisant à (4.21) a été démontrée dans la remarque 4.1. Donc, il existe deux constantes positives $\varepsilon_{G}$ et $L_{G}$ satisfaisant à (4.20). 
Etant établie (4.20), pour démontrer (4.14), on considère séparément les deux cas

i)

$$
\int_{D} A U(N) d x=\int_{D} \gamma(N) d x \leq-\frac{\varepsilon_{G} m e s(D)}{2},
$$

ii)

$$
\int_{D} A U(N) d x=\int_{D} \gamma(N) d x>-\frac{\varepsilon_{G} m e s(D)}{2} .
$$

Dans le cas $i$ ), on a évidemment

$$
G_{2}(N) \leq \int_{D} U(N) d x \int_{D} A U(N) d x \leq-\frac{\varepsilon_{G} m e s(D)}{2} \int_{D} U(N) d x,
$$

d'où résultent (4.13)-(4.14).

Dans le cas $i i$ ), en utilisant la notation $\bar{\Gamma}$ donnée dans (4.18), on obtient

$$
\begin{aligned}
-\frac{\varepsilon_{G}}{2} \text { mes }(D) \leq & \left.\left.\int \sum_{i=1}^{2}\left|\log N_{i}\right|>L_{G}\right\} \quad \gamma(N) d x+\int \sum_{i=1}^{2}\left|\log N_{i}\right| \leq L_{G}\right\} \\
& \leq-\varepsilon_{G} \operatorname{mes}\left\{\sum_{i=1}^{2}\left|\log N_{i}\right|>L_{G}\right\}+\bar{\Gamma} \operatorname{mes}\left\{\sum_{i=1}^{2}\left|\log N_{i}\right| \leq L_{G}\right\} \\
& \leq-\varepsilon_{G} \operatorname{mes}(D)+\left(\bar{\Gamma}+\varepsilon_{G}\right) \operatorname{mes}\left\{\sum_{i=1}^{2}\left|\log N_{i}\right| \leq L_{G}\right\},
\end{aligned}
$$

d'où on obtient

$$
\operatorname{mes}\left\{\sum_{i=1}^{2}\left|\log N_{i}\right| \leq L_{G}\right\} \geq \frac{\varepsilon_{G} m e s(D)}{2\left(\varepsilon_{G}+\bar{\Gamma}\right)}>0 .
$$

En vertu de l'inégalité de Poincaré il existe une constante positive $C^{\prime}$, qui dépend de $D$ et de $\frac{\varepsilon_{G} m e s(D)}{2\left(\varepsilon_{G}+\bar{\Gamma}\right)}$ et telle que

$$
\begin{aligned}
& \sum_{i=1}^{2}\left\|\log N_{i}\right\|_{L^{1}(D)}- \operatorname{mes}(D) L_{G} \\
& \leq \int_{D}\left(\sum_{i=1}^{2}\left|\log N_{i}\right|-L_{G}\right)^{+} d x \leq C^{\prime} \sum_{i=1}^{2}\left\|\nabla \log N_{i}\right\|_{L^{2}(D)} .
\end{aligned}
$$

Donc, compte tenu de

$$
\int_{D} A U(N) d x=\int_{D} \gamma(N) d x \leq \bar{\Gamma} \operatorname{mes}(D),
$$


on a

$$
\begin{aligned}
\int_{D} A U(N) d x-\frac{1}{2} \sum_{i=1}^{2} \varepsilon_{i}\left\|\nabla \log N_{i}\right\|_{L^{2}(D)} & \rightarrow-\infty \\
& \operatorname{pour} \sum_{i=1}^{2}\left\|\log N_{i}\right\|_{L^{1}(D)} \rightarrow \infty .
\end{aligned}
$$

On remarque que dans le cas où $\sum_{i=1}^{2}\left|N_{i}\right|$ est suffisamment grand, grâce à (4.17) l'inégalité du cas $i$ ) est vérifiée. Donc, en rappelant (4.4) et (4.19), des considérations sur le cas $i$ ) et de la relation (4.22) on déduit (4.13)-(4.14). Le lemme est démontré.

LEMME 4.2. Si on pose

$$
G(N)=G_{1}(N)+G_{2}(N)+G_{3}(N)
$$

$\left(G_{i}(N), i=1,2,3\right.$, étant définies dans (4.8)-(4.11)), alors $G(N)$ vérifie la condition (3.6).

DÉmonstration. On remarque que, comme

$$
\left\|N_{i}\right\|_{L^{2}(D)}^{3} \leq(\operatorname{mes}(D))^{1 / 2} \int_{D} N_{i}^{3} d x
$$

on a

$$
-\int_{D} \sum_{i, j=1}^{2} \beta_{i j} N_{i}^{2} N_{j} d x \leq-c_{1}\left(\sum_{i=1}^{2}\left\|N_{i}\right\|_{L^{2}(D)}^{2}\right)^{3 / 2}
$$

avec une constante positive $c_{1}$.

En outre, on a

$$
\begin{aligned}
\int_{D} \frac{\partial U}{\partial N_{i}} \rho_{i} N_{i} \lambda_{m} e_{m} d x \int_{D} \frac{\partial U}{\partial N_{j}} \rho_{j} N_{j} \lambda_{m} e_{m} d x \\
=\int_{D} \rho_{i}\left(k_{i} N_{i}-1\right) \lambda_{m} e_{m} d x \int_{D} \rho_{j}\left(k_{j} N_{j}-1\right) \lambda_{m} e_{m} d x \\
\leq \frac{1}{2} \int_{D} \lambda_{m}^{2} e_{m}^{2} d x\left(\rho_{i}^{2} \int_{D}\left(k_{i} N_{i}-1\right)^{2} d x+\rho_{j}^{2} \int_{D}\left(k_{j} N_{j}-1\right)^{2} d x\right) .
\end{aligned}
$$


En rappelant (4.11) et en tenant compte de la condition (2.5), on obtient

$$
G_{3}(N) \leq c_{2} K_{0}\left[\int_{D}\left(k_{1} N_{1}-1\right)^{2} d x+\int_{D}\left(k_{2} N_{2}-1\right)^{2} d x\right]
$$

avec une constante $c_{2}$. Donc, en rappelant (4.8) on a

$$
G_{1}(N)+G_{3}(N)+\int_{D} \sum_{i, j=1}^{2} \beta_{i j} N_{i}^{2} N_{j} d x \leq c_{3} \sum_{i=1}^{2}\left\|N_{i}\right\|_{L^{2}(D)}^{2}+c_{4}
$$

avec deux constantes $c_{3}$ et $c_{4}$. Par conséquent, compte tenu de (4.23), on a

$$
\sup _{N \in \Xi(D)}\left(G_{1}(N)+G_{3}(N)\right)<\infty
$$

$$
\begin{array}{r}
\sup \left\{G_{1}(N)+G_{3}(N) \mid N \in \Xi(D), \sum_{i=1}^{2}\left\|N_{i}\right\|_{L^{2}(D)}^{2} \geq c\right\} \rightarrow-\infty \\
\text { pour } c \rightarrow+\infty .
\end{array}
$$

Du lemme 4.1 et des relations (3.1), (4.24), (4.25), on déduit (3.6).

DÉMONSTRATION DU LEMME 3.1. On remarque que par la propriété des martingales, on a

$$
\boldsymbol{E} \int_{0}^{t}\left\langle h\left(N\left(t^{\prime}, \cdot\right)\right), d W\left(t^{\prime}\right)\right\rangle=0
$$

On remarque d'autre part que les définitions de $\Phi_{0}(N), \Phi_{1}(N), \varphi(N)$, $U(N), F(N)$ (voir aussi (3.8), (4.2), (4.3), (4.4)) impliquent qu'il existe des constantes positives $\kappa_{2}, C_{0}, C_{1}$ telles que

$$
\Phi_{0}(N) \leq C_{0} \varphi(N) \quad \forall N \in \Xi(D),
$$

$$
\begin{gathered}
\Phi_{1}(N) \leq C_{1} F(N) \quad \forall N \in\left\{N \in \Xi(D) \mid \nabla N_{i}, \nabla \log N_{i} \in L^{2}(D), i=1,2\right\}, \\
\varphi(N) \leq \kappa_{2}\|N\|_{\Xi}^{2} .
\end{gathered}
$$

Compte tenu de (4.27), (4.28) et (4.29) et en posant $\kappa_{0}=\frac{1}{C_{0}}, \kappa_{1}=\frac{1}{C_{1}}$, le lemme 3.1 résulte de (4.6) et du lemme 4.2. 


\section{BIBLIOGRAPHIE}

[1] A. C. CaPelo, Modelli matematici in biologia . Decibel editrice, Padova, 1989.

[2] S. Chessa - H. Fujita Yashima, Equazione stocastica di dinamica di popolazioni di tipo preda-predatore. Boll. U.M.I., Serie VIII, vol. 5 - B (2002), pp. 789-804.

[3] F. B. Christiansen - T. M. Fenchel, Theories of population in biological communities. Springer, 1977.

[4] G. Da Prato, An introduction to infinite dimensional analysis. Scuola Norm. Sup. Pisa, 2001.

[5] G. Da Prato - J. ZABCZYK, Ergodicity for infinite dimensional systems. Cambridge Univ. Press, 1996.

[6] H. Fujita Yashima, Equation stochastique de dynamique de populations du type proie-prédateur avec diffusion dans un territoire. Novi Sad J. Math., vol. 33 (2003), pp. 31-52.

[7] H. Fujita Yashima - S. Hamdous, Mesure invariante pour l'équation stochastique d'un modèle de compétition limitée entre des espèces avec une diffusion spatiale. Rend. Circ. Mat. Palermo, Serie II, Tomo 56 (2007), pp. 79-89.

[8] B. GabUti - A. Negro, Some results on asymptotic behaviour of the Volterralotka diffusion equations. Rend. Sem. Math. Univ. Polit., Torino, vol. 36 (1977/ 1978), pp. 403-414.

[9] I. I. Guikhman - A. V. Skorokhod, Introduction à la théorie des processus aléatoires (traduit du russe). Mir (Moscou), 1980.

[10] R. Z. HaS'MinskiI, Stochastic stability of differential equations . (translated from Russian). Sijthoff \& Noordhoff, Alphe ann den Rijn, 1980.

[11] N. Kryloff - N. Bogoliuboff, La théorie générale de la mesure dans son application à l'étude des systèmes dynamiques de la mécanique non linéaire. Annals Math., vol. 38 (1937), pp. 65-113.

[12] A. Negro - B. Gabuti, A fractional steps method for the Volterra-lotka diffusion equations. Rend. Sem. Math. Univ. Polit., Torino, vol. 35 (1976/ 1977), pp. 373-389.

[13] E. PARDOux, Intégrales stochastiques hilbertiennes. Publication interne Univ. Paris - Dauphine, 1976.

[14] R. RUDNICKI, Long-time behaviour of a stochastic prey-predator model. Stoch. Proc. Appl., vol. 108 (2003), pp. 93-107.

[15] E. Tornatore, Stochastic equation of population dynamic with diffusion on a domain. Rend. Circ. Mat. Palermo, Serie II, Tomo 52 (2003), pp. 15-29.

[16] E. Tornatore - L. Manca - H. Fujita Yashima, Comportamento asintotico della soluzione del sistema di equazioni stocastiche per due specie in competizione. Rend. Ist. Lombardo Accad. Sci. Lett. (Sci. Mat. Appl.). vol. 136/137 (2002/03), pp. 151-183.

[17] V. Volterra, Leçons sur la théorie mathématique de la lutte pour la vie. Gauthier-Villars, Paris, 1931.

Manoscritto pervenuto in redazione il 16 luglio 2008. 